\title{
Proctocolitis caused by lymphogranuloma venereum
}

\author{
Edward Weiss MD, Marie Sano BScN RN
}

Cite as: CMAJ 2018 March 19;190:E331-3. doi: 10.1503/cmaj.171292

A 47-year-old man was referred to our anal dysplasia clinic for the evaluation of ongoing anorectal discomfort and an anal mass. The patient had experienced severe rectal pain and cramping and incomplete defecation, and had passed stools containing pus and blood. He also had intermittent fevers and difficulty starting and maintaining urine flow. These symptoms had caused him considerable distress and disruption to his daily life over the previous four months.

The patient's medical history included HIV infection, for which he was receiving stable antiretroviral therapy. He also had a history of coronary artery disease, with two previous myocardial infarctions requiring percutaneous intervention. The rest of his medical history was noncontributory. The patient was in a stable but nonmonogamous relationship with another man and practised anal receptive intercourse with inconsistent condom use.

The patient's symptoms were initially attributed to external hemorrhoids. When his condition did not improve with usual hemorrhoid treatment, he presented to the emergency department, where he underwent computer tomography (CT) imaging of the abdomen and pelvis (Figure 1) that showed moderate mucosal edema involving the sigmoid colon. The interpreting radiologist suggested that "it could represent an inflammatory process ... or infectious colitis." The patient was referred to an outpatient endoscopy clinic for further investigation where he underwent a colonoscopy. The endoscopist noted the use of copious irrigation to ensure visibility. The only finding of note was an "anal mass," which was biopsied. Histology from the lesion was reported as "acute inflammatory exudate," as well as "a small fragment of colonic mucosa showing low-grade dysplastic change."

Before being seen at our clinic, the patient underwent urine nucleic acid testing for gonorrhea and chlamydia, the results of which were negative. Screening for syphilis and hepatitis $C$ was also negative. A culture of the patient's stool showed no bacterial infection; Blastocystis hominis grew on culture, but the patient was not given treatment for it. Other laboratory investigations included an undetectable HIV RNA target and a CD4 count of 1129 cells $/ \mathrm{mm}^{3}$, with a CD4 percentage of 27.1 and a CD4:CD8 ratio of 0.7.

On physical examination, the patient had diffuse abdominal tenderness and no obvious inguinal lymphadenopathy. His perianal skin was normal. A digital rectal examination caused extreme pain and did not suggest any obvious mass. Upon insertion of an

\section{KEY POINTS}

- Clinicians should suspect lymphogranuloma venereum proctitis when a patient presents with typical symptoms (rectal pain, abdominal cramping, discharge and bleeding ) and risk factors for anal chlamydia transmission, such as HIV infection, or a history of anal intercourse or same-sex male intercourse.

- Nucleic acid testing is more sensitive than chlamydial culture for detecting infection, but may not be available in all settings.

- Urine nucleic acid amplification tests alone are not sufficient, because a substantial proportion of chlamydial infections in men who have sex with men will be extragenital.

- Empiric treatment with doxycycline should be considered if the index of suspicion for lymphogranuloma venereum proctitis is high.

anoscope, we saw florid proctitis with purulent discharge and friable anal and rectal mucosa (Figure 2). We took swabs from the most purulent areas for chlamydial and gonorrheal culture. We opted to defer a high-resolution anoscopy owing to the patient's acutely abnormal mucosa and his discomfort.

Based on the suggestive history and examination, we considered a diagnosis of lymphogranuloma venereum to be the most likely cause of the patient's symptoms, which was treated empirically with a 21-day course of doxycycline (100 mg taken orally, twice daily). Cultures for Chlamydia species showed growth, and molecular testing confirmed the presence of Chlamydia trachomatis serovar L2b, a known cause of lymphogranuloma venereum.

The patient reported complete resolution of his symptoms after completing the course of doxycycline. He has regular follow-up with high resolution anoscopy for unrelated findings of anal dysplasia.

\section{Discussion}

Lymphogranuloma venereum is a sexually transmitted infection associated with invasive serovars L1, L2 and L3 of C. trachomatis. ${ }^{1}$ The infection has historically been associated with painful inguinal lymphadenopathy (buboes) as its pathognomonic sign; however, in recent years, increasing attention has been given to rectal presentations of lymphogranuloma venereum. ${ }^{2}$ Inoculation of chlamydial pathogens in the rectal mucosa, rather than the penile or vulvar 
tissue, can result in a severe hemorrhagic proctitis or proctocolitis without the development of clinically apparent lymphadenopathy. The clinical appearance can often mimic that of inflammatory bowel disease, and the infection may not be suspected unless there is no response to treatment or further inquiries are made into the patient's sexual practices or HIV status.

Lymphogranuloma venereum has been portrayed as an infection not commonly encountered or transmitted in developed countries. However, starting in the early 2000s, case reports of the infection among populations of men who have sex with men in northern and western Europe grew in frequency. ${ }^{1}$ Recent statistics have shown a substantial increase in the incidence of lymphogranuloma venereum in North America. Between 2004 and 2012, before national surveillance for lymphogranuloma venereum was discontinued, 170 cases were reported to the Public Health Agency of Canada; ${ }^{3}$ in 2016, 39 cases were reported in Toronto alone in the first six months of the year. ${ }^{4}$ Of these latter cases, all of the patients were men, and $48 \%$ involved HIV coinfection.

The natural history of lymphogranuloma venereum infection is generally divided into three stages. Patients may initially have a

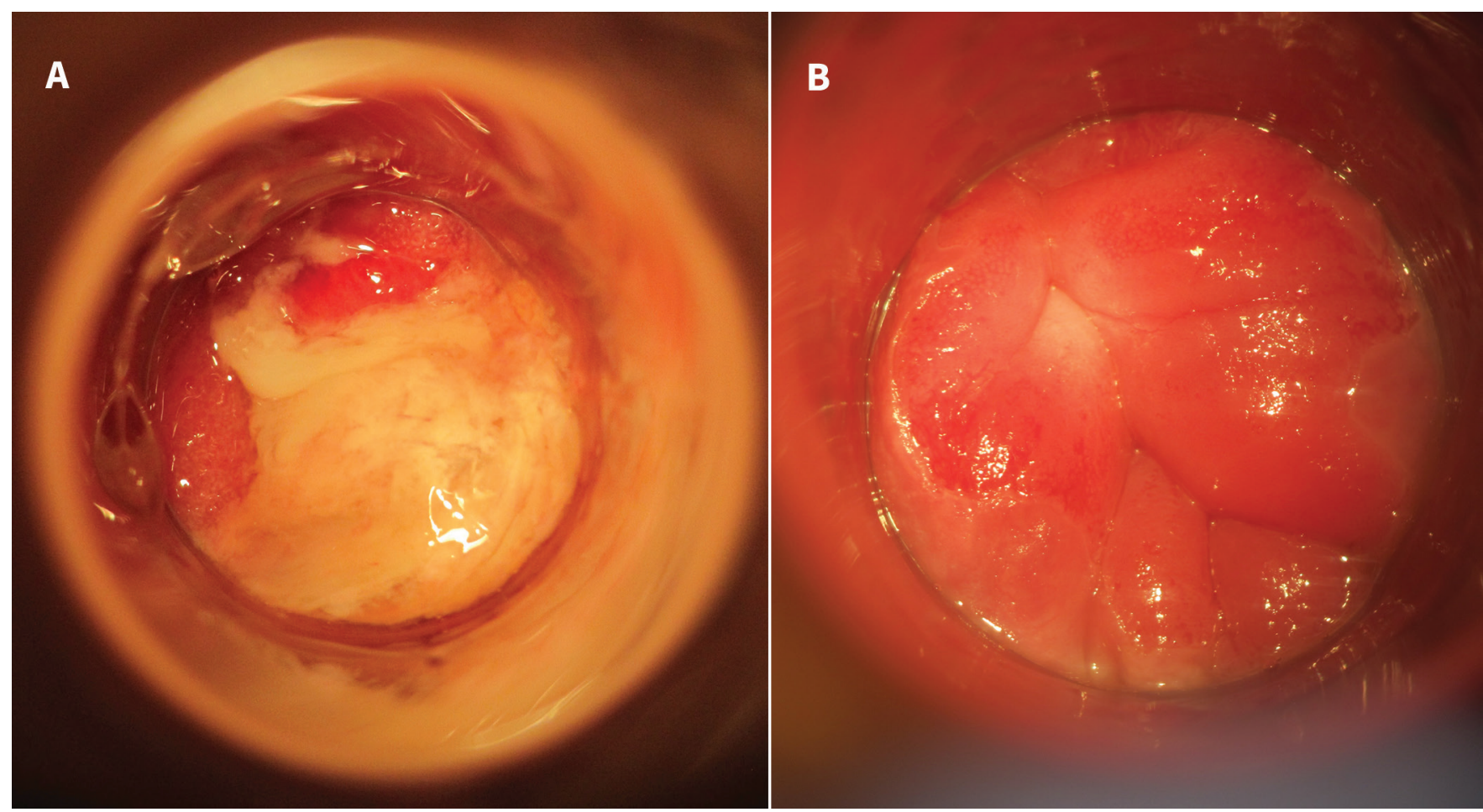

Figure 1: Anoscopic image of the anorectal junction showing diffuse purulent proctitis before treatment (A) and restoration of normal anatomy six months later (B) in a 47-year-old man referred to an anal dysplasia clinic for the evaluation of ongoing anorectal discomfort and an anal mass.
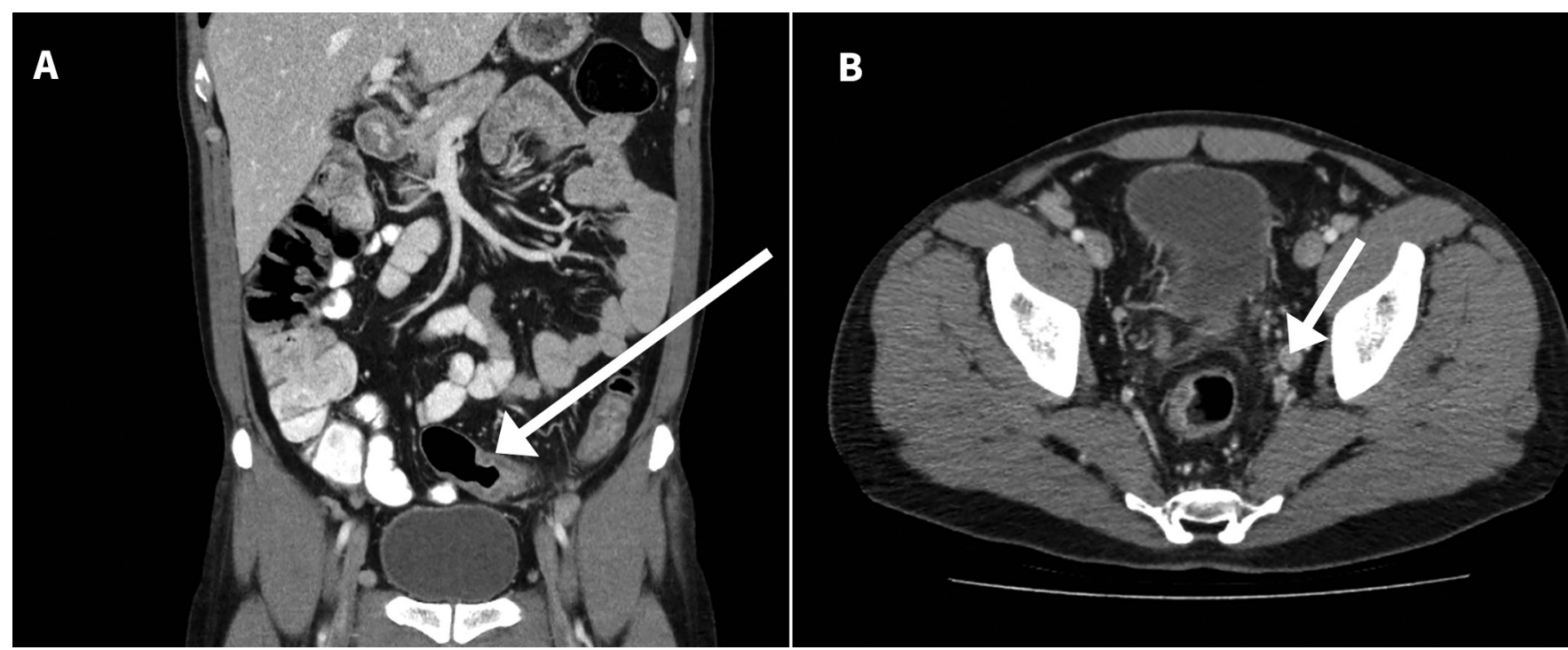

Figure 2: Contrast-enhanced computed tomography image of the patient's abdomen and pelvis showing thickened mucosa of the sigmoid colon (A, arrow) and an enlarged obturator lymph node (B, arrow). 
self-limited painless papule in the genital mucosa or in the rectum, which can often go unnoticed. Within six weeks of the initial lesion, secondary lymphogranuloma venereum manifests with either a florid and painful inguinal or femoral lymphadenopathy, or an acute hemorrhagic proctitis. Patients may have rectal discharge or pain, feel an urge to defecate or incomplete defecation, or have rectal bleeding and anal itching. Systemic symptoms such as fevers, chills or malaise are common. Finally, tertiary lymphogranuloma venereum may present with chronic inflammatory changes, such as lymphatic obstruction causing genital elephantiasis. ${ }^{1}$

Untreated lymphogranuloma venereum proctitis can lead to complications such as anorectal strictures and fistulae, which can cause substantial morbidity even after treatment has been completed. Complete bowel obstruction is uncommon, but is a recognized cause of death in severe cases. ${ }^{5}$ Delays in treatment are common, and multiple investigations are often undertaken before the correct diagnosis is made. We have seen several patients in our clinic who have undergone assessment by multiple specialists with unnecessary endoscopy and cross-sectional CT or magnetic resonance imaging before lymphogranuloma venereum was considered as a cause for their symptoms.

The diagnosis of lymphogranuloma venereum proctitis has previously been hampered by the lack of suitably sensitive testing methods. Traditional chlamydial culture techniques have limited sensitivity (in the range of 30\%-50\%), especially when performed blindly, without anoscopic guidance. ${ }^{6}$ However, nucleic acid amplification tests are much more sensitive in their ability to detect $C$. trachomatis. ${ }^{7}$ Although none of the commercial test kits available in Canada are approved for extragenital use, many laboratories have conducted independent validation of nucleic acid amplification testing for extragenital sites. Thus, it is important to check with local public health and laboratory authorities to determine availability of nucleic acid amplification testing for Chlamydia spp. (Alberto Severini, Viral Exanthemata and STD Section, National Microbiology Laboratory, Public Health Agency of Canada, Ottawa: personal communication, 2017). At the time of our patient's presentation, our hospital had not yet validated a suitable assay. Clinicians should be aware that urine nucleic acid amplification tests alone are not sufficient, because a substantial proportion of chlamydial infections in men who have sex with men will be extragenital. ${ }^{8}$

If lymphogranuloma venereum is suspected based on risk factors and clinical presentation, specimens that test positive for Chlamydia should be sent to the National Microbiology Laboratory (Winnipeg, Manitoba) for confirmatory serotyping by DNA sequencing. Serologic testing for lymphogranuloma venereum is not recommended owing to cross-reactions with other Chlamydia species and difficulties in interpreting variations in titres - specifically, low titres do not rule out lymphogranuloma venereum. ${ }^{9}$

Treatment is traditionally $100 \mathrm{mg}$ doxycycline twice daily for 21 days. Erythromycin is an alternative, and some authorities suggest weekly azithromycin for three weeks may be effective. ${ }^{1}$ Sexual partners should undergo treatment as per usual recommendations, with either azithromycin or doxycycline, even in the absence of symptoms. ${ }^{1}$ Testing for common coinfections, including HIV, gonorrhea, syphilis and hepatitis $\mathrm{C}$, is recommended, particularly for patients whose HIV status is not known ${ }^{1}$; empiric treatment for gonorrhea with ceftriaxone ( $250 \mathrm{mg}$ intramuscularly) should be considered at the time of presentation if exudate is present but other symptoms are not typical for lymphogranuloma venereum.

\section{Conclusion}

Lymphogranuloma venereum proctitis is a serious clinical entity that is increasing in incidence. Complications and prolonged symptoms can be avoided through the prudent and early collection of targeted rectal specimens, and by reviewing a thorough sexual health history of patients presenting with typical symptoms. Maintaining a high index of suspicion is necessary, and empiric treatment may be warranted while waiting for the results of investigations to avoid unnecessary morbidity.

\section{References}

1. Interim statement on the diagnosis, treatment and reporting of lymphogranuloma venereum (LGV) in Canada. Ottawa: Public Health Agency of Canada; modified 2005 June 6. Available: www.phac-aspc.gc.ca/publicat/lgv/lgv-rdt1-eng.php (accessed 2017 Apr. 24).

2. Weir E. Lymphogranuloma venereum in the differential diagnosis of proctitis. CMAJ 2005;172:185.

3. Totten S, MacLean R, Payne E, et al. Chlamydia and lymphogranuloma venereum in Canada: 2003-2012 - summary report. Can Commun Dis Rep 2015; 41-02:20.

4. Syphilis and lymphogranuloma venereum resurgence. Toronto: City of Toronto; updated 2017 Aug. 3. Available: www.toronto.ca/community-people/ health-wellness-care/information-for-healthcare-professionals/sexual-health -info-for-health-professionals/syphilis-and-lymphogranuloma-venereum -resurgence/ (accessed 2017 Apr. 24).

5. Richardson D, Goldmeier D. Lymphogranuloma venereum: an emerging cause of proctitis in men who have sex with men. Int J STD AIDS 2007;18:11-4, quiz 15.

6. Centers for Disease Control and Prevention. Recommendations for the laboratory-based detection of Chlamydia trachomatis and Neisseria gonorrhoeae - 2014. MMWR Recomm Rep 2014;63:1-19.

7. Ceovic R, Gulin SJ. Lymphogranuloma venereum: diagnostic and treatment challenges. Infect Drug Resist 2015;8:39-47.

8. Kent CK, Chaw JK, Wong W, et al. Prevalence of rectal, urethral, and pharyngeal chlamydia and gonorrhea detected in 2 clinical settings among men who have sex with men: San Francisco, California, 2003. Clin Infect Dis 2005;41:67-74

9. Supplementary statement concerning the laboratory diagnosis of lymphogranuloma venereum (LGV): Canadian guidelines on sexually transmitted infections. Ottawa: Public Health Agency of Canada; 2014. Available: www. canada.ca/content/dam/phac-aspc/migration/phac-aspc/std-mts/sti-its/cgsti -ldcits/assets/pdf/appendix-supp-lgv-eng.pdf (accessed 2017 Nov. 16).

\section{Competing interests: None declared.}

This article has been peer reviewed.

The authors have obtained patient consent.

Affiliation: Immunodeficiency Clinic, Department of Medicine, University Health Network, Toronto, Ont.
Contributors: Edward Weiss participated in direct clinical care of the patient and drafted the article. Marie Sano provided direct clinical care of the patient and helped revise the article. Both authors approved the final version of the article to be published and agreed to be responsible for its integrity.
Acknowledgements: The authors thank Dr. Irving Salit for his guidance and support, and Dr. William Magnuson for his assistance with the computed tomography images.

Correspondence to: Edward Weiss, edward.weiss@uhn.ca 